\title{
Nonlinear anisotropic diffusion filtering of three- dimensional image data from two-photon microscopy
}

\author{
Philip. J. Broser \\ Max-Planck-Institut für Medizinische Forschung \\ Abteilung Zellphysiologie \\ Jahnstr. 29 \\ D-69120 Heidelberg, Germany \\ and \\ Universität Heidelberg \\ Ifl-Simulation in Technology \\ Im Neuenheimer Feld 368 \\ D-69120 Heidelberg, Germany \\ E-mail: philip.broser@mpimf-heidelberg.mpg.de
}

\section{R. Schulte}

S. Lang

Universität Heidelberg

Ifl-Simulation in Technology

Im Neuenheimer Feld 368

D-69120 Heidelberg, Germany

\section{A. Roth}

\section{Fritjof Helmchen}

J. Waters

Max-Planck-Institut für Medizinische Forschung

Abteilung Zellphysiologie

Jahnstr. 29

D-69120 Heidelberg, Germany

\section{Bert Sakmann}

Max-Planck-Institut für Medizinische Forschung

Abteilung Zellphysiologie

Jahnstr. 29

D-69120 Heidelberg, Germany

\section{G. Wittum}

Universität Heidelberg

IfI-Simulation in Technology

Im Neuenheimer Feld 368

D-69120 Heidelberg, Germany

\begin{abstract}
Two-photon microscopy in combination with novel fluorescent labeling techniques enables imaging of three-dimensional neuronal morphologies in intact brain tissue. In principle it is now possible to automatically reconstruct the dendritic branching patterns of neurons from 3-D fluorescence image stacks. In practice however, the signal-to-noise ratio can be low, in particular in the case of thin dendrites or axons imaged relatively deep in the tissue. Here we present a nonlinear anisotropic diffusion filter that enhances the signal-to-noise ratio while preserving the original dimensions of the structural elements. The key idea is to use structural information in the raw datathe local moments of inertia-to locally control the strength and direction of diffusion filtering. A cylindrical dendrite, for example, is effectively smoothed only parallel to its longitudinal axis, not perpendicular to it. This is demonstrated for artificial data as well as for in vivo two-photon microscopic data from pyramidal neurons of rat neocortex. In both cases noise is averaged out along the dendrites, leading to bridging of apparent gaps, while dendritic diameters are not affected. The filter is a valuable general tool for smoothing cellular processes and is well suited for preparing data for subsequent image segmentation and neuron reconstruction. $\odot 2004$ Society of Photo-Optical Instrumentation Engineers. [DOI: 10.1117/1.1806832]
\end{abstract}

Keywords: filters; diffusion; nonlinear; anisotropic; two-photon imaging; neural morphology; reconstruction.

Paper 04008 received Jan. 27, 2004; revised manuscript received May 10, 2004; accepted for publication May 10, 2004.

\section{Introduction}

Two-photon laser scanning microscopy ${ }^{1}$ has become a principal technique for high-resolution fluorescence imaging in various biological tissues because it provides intrinsic optical sectioning and exceptional depth penetration (for reviews see Refs. 2, 3, and 4). Imaging depths in the cortex of more than $500 \mu \mathrm{m}$ are now routinely achieved and image acquisition from $1 \mathrm{~mm}$ inside a mouse neocortex has been demonstrated recently. ${ }^{5}$ Combined with techniques for labeling individual neurons or sparse populations of neurons, e.g., dye loading via intracellular pipettes ${ }^{6,7}$ or the expression of fluorescent proteins, ${ }^{8-10}$ two-photon microscopy can resolve neurons with high resolution in vivo, i.e., within the intact brain of living animals. ${ }^{6,11}$ Thus, 3-D fluorescence images of neurons can be obtained including their entire dendritic morphology within their native environment.

Address all correspondence to Philip Julian Broser, Max-Planck-Institut für medizinische Forschung, Jahnstr. 29, D-69120 Heidelberg, Germany. Tel: 49172-6783108; Fax: 49-6221-486-459; E-mail: philip.broser@mpimfheidelberg.mpg.de

1083-3668/2004/\$15.00 @ 2004 SPIE
These advances in imaging technology are prerequisites for the automatic reconstruction of neuronal morphologies. An automatic reconstruction would allow fast, highthroughput determination of characteristic anatomical features, for instance the dendritic branching pattern of different neuronal cell types. This is in contrast to standard manual reconstruction techniques, which are time-consuming and highly dependent on the experience of the human anatomist. ${ }^{12}$ They also suffer from scaling problems due to shrinkage in fixed tissue. Automatic reconstruction would furthermore help to establish large databases of neuronal morphologies for biophysical modeling of cellular and neural network signal processing.

A number of different approaches for automatically reconstructing neuronal morphologies have been reported previously. ${ }^{13-15}$ Recently, Maravall et al. ${ }^{16}$ automatically reconstructed a large number of layer $2 / 3$ pyramidal neurons for an analysis of experience-dependent plasticity of their dendritic branching pattern. However, results in general strongly depend on the quality of the image data and in most cases require preprocessing. One of the major obstacles for devel- 

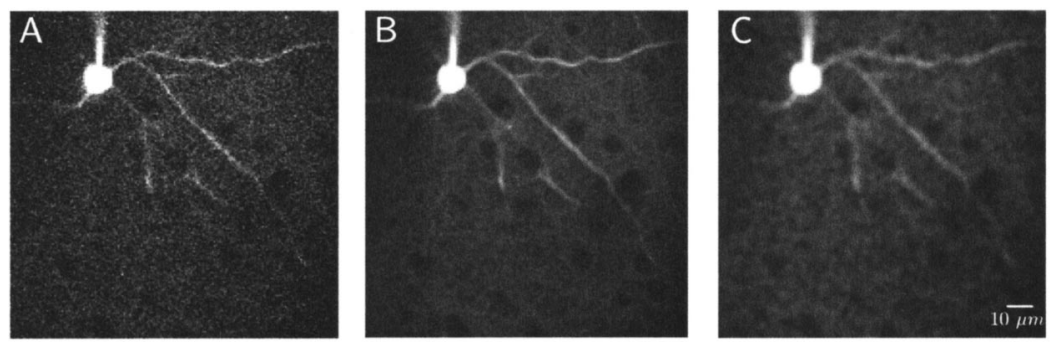

Fig. 1 Comparison of the anisotropic diffusion filtering with Gaussian blur. (a) One slice of a data stack from a two-photon scan of a pyramidal cell in layer $2 / 3$ of rat somatosensory cortex in vivo. This slice is at a depth of $280 \mu \mathrm{m}$ inside the brain. The loading pipette is visible at the top of the image next to the cell body. (b) Filtering with anisotropic diffusion. The filter was used with two time steps, a time step size of $\tau=2.0$ and a scanning range of $\delta=10 \mu \mathrm{m}$. (c) Data after Gaussian blur with $\sigma=2.8 \approx 2 \sqrt{(2)}$ (measured in voxel).

oping an automatic reconstruction algorithm is the noise inherent in low-level fluorescence images. For example, using two-photon microscopy for in vivo imaging, both excitation light and fluorescence light are increasingly scattered with imaging depth, causing a reduction in signal-to-noise ratio and making it difficult to fully resolve thin, weakly fluorescent neural processes [Fig. 1(a)]. As a result, simple thresholding procedures for image segmentation might erroneously insert gaps into dendritic branches, preventing the reconstruction of a fully connected dendritic tree. Therefore, preprocessing of the raw fluorescence data in order to increase the signal-to-noise ratio while preserving dendritic structure is an essential prerequisite for automatic segmentation and subsequent morphological reconstruction.

One way to preprocess the raw data is filtering. A wide range of filters exist in image processing. The most basic filters calculate an average brightness value in a region around a central voxel. Other, more sophisticated filters use spectral analysis to extract signals within a defined bandwidth, such as low- or high-pass filters. Both methods show a close connection to the theory of partial differential equations. ${ }^{17}$ Actually the well-known Gaussian blur is an excellent low-pass filter. ${ }^{18}$ But none of these methods are sensitive to the local structure of the processed data.

One of the first approaches to include information about the data into the filter was made by the direction-pyramidal decomposition method. ${ }^{19}$ Alternatively, wavelet shrinkage has been used for preprocessing neuronal image data. ${ }^{20,21}$ One of the advantages of the wavelet approach is the use of multiple scales. A shortcoming is that the filtering threshold in the wavelet space cannot be determined automatically. One of the reasons is that the proper threshold depends on the local noise statistic within the image. Because this threshold varies throughout the data set an optimal value is difficult to find and thus no optimal denoising strategy can be given in general. A more general way to take the data structure into account is the use of diffusion filters which have a long tradition in image processing. ${ }^{17}$ They have mostly been used as convolution filters like Gaussian blurring. Nonlinear diffusion filters were first used by Perona and Malik in $1987 .{ }^{22}$ Since then many specific filters have been used to address a wide range of problems. For instance, an anisotropic diffusion filter controlled by local properties of the data was used by Lenzen ${ }^{23}$ to reconstruct DNA structures.

In our case the filter has to prepare the data for segmentation. The filtering process aims to (1) separate noise and sig- nal, (2) close apparent gaps in the structure, and (3) preserve dendritic diameters. For this purpose we designed an anisotropic diffusion filter which is now implemented in our software toolbox, NEURA (NEUron Reconstruction Algorithm). Here, we present this diffusion filter designed specifically for three-dimensional data of nerve cells. The filter is tuned to delete disturbances (remove noise) and to bridge open structures while preserving dendritic diameters. In this respect, it performs much better than Gaussian smoothing (see Fig. 1 for a first impression). The primary goal of our algorithm is to facilitate automatic reconstruction of neuronal morphology, for example in order to import them into the NEURON simulation environment ${ }^{24}$ for in silico experiments.

\section{Material and Methods}

\subsection{Linear Isotropic Diffusion and Gaussian Blur}

Linear isotropic diffusion is described by the partial differential equation (pde):

$$
\begin{gathered}
\partial_{t} u(x, t)=\Delta u(x, t), x \in \mathbb{R}^{n} \\
u(x, 0)=u_{0}(x) \text { on } \mathbb{R}^{n}
\end{gathered}
$$

The solution tends to zero for $t \rightarrow \infty$. In image processing the time $t$ is an artificial parameter. In case of linear diffusion the filter makes sense only if the time is limited to a finite value as can be seen comparing linear diffusion with a simple Gaussian blur. Gaussian blur or Gaussian smoothing is an excellent low-pass filter in image processing. It attenuates high frequencies in a monotonic way. ${ }^{18}$ The close connection between linear diffusion and Gaussian blur gives a deeper understanding of the filter process.

Let a gray-scale image $u$ be represented by a real-valued mapping $u_{0}(x) \in L^{1}\left(\mathbb{R}^{n}\right)$. The linear diffusion process (1) can be solved analytically for any time $t>0$ by using the Green's function for the diffusion equation, which is actually the Gaussian kernel ${ }^{25}$ :

$$
u(x, t)=\int_{\mathbb{R}^{n}} u_{0}(y) \cdot \frac{1}{(4 \pi t)^{n / 2}} e^{-(x-y)^{2} / 4 t} \mathrm{~d} y .
$$

The Gaussian smoothing of $u_{0}$ is described by: 


$$
\begin{aligned}
u(x, \sigma) & =\left(u_{0} * G_{\sigma}\right)(x) \\
& =\int_{\mathbb{R}^{n}} u_{0}(y) \cdot \frac{1}{\left(2 \pi \sigma^{2}\right)^{n / 2}} e^{-(x-y)^{2} / 2 \sigma^{2}} \mathrm{~d} y .
\end{aligned}
$$

Apparently the time $t$ has the same effect as the blurring parameter $\sigma$ (that means filtering an image stack with linear diffusion $t=2.0$ is the same as using a Gaussian blur with $\sigma^{2}=4.0$ ). For finite times a linear diffusion filter yields a smoothing of the picture, which is desirable to suppress noise on large homogeneous faces. On distinct structures like sharp edges, however, isotropic diffusion leads to undesirable blurring of the structure as illustrated in the examples below.

\subsection{Nonlinear Anisotropic Diffusion}

To avoid broadening of edges, while preserving the smoothing of uniform surfaces, we need an anisotropic diffusion operator which leads to isotropic diffusion on surfaces, but avoids cross-diffusion at sharp edges. This implies that the operator [see Eq. (1)] needs to depend on the structure of the image, i.e., it must be nonlinear. In 1987, Perona and Malik ${ }^{22}$ introduced such a nonlinear operator using the gradient of the data to control the diffusion. Their algorithm was designed specifically to preserve edges with diffusion occurring mainly perpendicular to the gradient of the image data in order to enhance edges in two dimensions. More recent results using a gradient-controlled approach can be found in Refs. 26 and 27.

Mathematically speaking, modeling a (nonlinear anisotropic) diffusion process means to solve the following boundary value problem:

$$
\begin{gathered}
\partial_{t} u=\nabla \cdot(D(u) \cdot \nabla u) \quad \text { on } \mathbb{R}^{+} \times \Omega \\
u(x, 0)=u_{0}(x) \quad \text { on } \bar{\Omega} \\
(D(u) \cdot \nabla u) \cdot \vec{n}=0 \quad \text { on } \quad \mathbb{R}^{+} \times \partial \Omega
\end{gathered}
$$

with the permeability tensor $D(u)$. In our case $\Omega \subset \mathrm{R}^{3}$ describes the image data volume, $u_{0}(x)$ the raw data set, and $\vec{n}$ the outer normal at the volume boundary $\partial \Omega$. The third equation states that no flux crosses the image boundary, i.e., no signal is lost from the image.

In the case of $D$ being the identity this is the linear isotropic diffusion, which we have already seen in Eq. (1). The choice of the tensor $D(u)$ is crucial for the performance of the filter. Note that time is no longer just a blurring parameter. Because of the nonlinearity $D(u)$ the solution does not necessarily tend to zero for $t \rightarrow \infty$; see Ref. 27. Instead a steady state solution of the equation might exist. To choose an appropriate permeability tensor $D(u)$ we need to extract structural information from the raw gray value data $u(x, t)$.

\subsection{Structure Detection}

Fluorescence images often are too noisy to use a gradient criterion to control the diffusion direction. More information is needed to reliably detect the object structure. In the case of filtering cellular processes, e.g., neuronal dendrites, we would like to use strong diffusion parallel to the main axis of the dendrite but not perpendicular to it. Thus we have to find a way to detect the axis of the dendrite locally. Motivated by Lenzen and Rumpf ${ }^{23}$ we decided to use the moments of inertia.

\subsubsection{Moment of inertia}

To determine the local structure of the data in a threedimensional image stack we consider the gray value function as a density function of a real body. Then we can calculate the (local) moments of the virtual body, by choosing an integration volume $B_{\delta}\left(x_{0}\right)$ around the voxel of interest. The parameter $\delta$ represents the size of the integration volume and is referred to as scanning range. ${ }^{28}$ The local moments are defined by:

- mass:

$$
M^{0}\left(x_{0}\right)=\int_{B_{\delta}\left(x_{0}\right)} u(x) \mathrm{d} x
$$

- center of mass:

$$
M^{1}\left(x_{0}\right)=\frac{1}{M^{0}\left(x_{0}\right)} \int_{B_{\delta}\left(x_{0}\right)} u(x) x \mathrm{~d} x,
$$

- moment of inertia:

$$
M^{2}\left(x_{0}\right)=\int_{B_{\delta}\left(x_{0}\right)} u(x)\left[x-M^{1}\left(x_{0}\right)\right] \otimes\left[x-M^{1}\left(x_{0}\right)\right] \mathrm{d} x,
$$

with $\otimes$ being the outer product.

The eigenvectors of the moment of inertia are the main axes of inertia. The eigenvalues contain information about the spatial structure. The size of the integration volume, the scanning range, is a critical parameter (see below).

\subsubsection{Example}

The eigenvectors and eigenvalues of the moment of inertia of a hexahedron (see Fig. 2) can easily be calculated. The tensor calculated at the origin is:

$$
M^{2}(x)=\frac{1}{12}\left(\begin{array}{ccc}
a^{3} b c & 0 & 0 \\
0 & a b^{3} c & 0 \\
0 & 0 & a b c^{3}
\end{array}\right) .
$$

The corresponding eigenvectors and the eigenvalues are given by:

$$
\begin{gathered}
V_{1}(0)=\left(\begin{array}{l}
1 \\
0 \\
0
\end{array}\right), \quad V_{2}(0)=\left(\begin{array}{l}
0 \\
1 \\
0
\end{array}\right), V_{3}(0)=\left(\begin{array}{l}
0 \\
0 \\
1
\end{array}\right), \\
\alpha_{1}=\frac{1}{12} a^{3} b c, \quad \alpha_{2}=\frac{1}{12} a b^{3} c, \quad \alpha_{3}=\frac{1}{12} a b c^{3}
\end{gathered}
$$

\subsubsection{Geometry classification}

Following Lenzen ${ }^{23}$ we define the following variables to quantify the size of the eigenvalues:

$$
c_{1}=\frac{\alpha_{1}-\alpha_{2}}{\sum \alpha_{i}}, \quad c_{2}=\frac{2\left(\alpha_{2}-\alpha_{3}\right)}{\sum \alpha_{i}} \quad \text { and } \quad c_{3}=\frac{3 \alpha_{3}}{\sum \alpha_{i}} \text {, }
$$




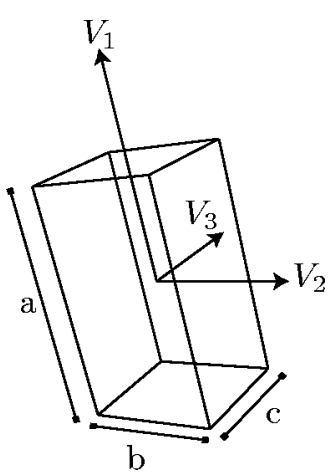

Fig. 2 Direction of the eigenvectors of the moment of inertia of a hexahedron. The structure is assumed to be solid with a constant density function. $\mathrm{a}, \mathrm{b}, \mathrm{c}$ : the three sides, $V_{1}, V_{2}, V_{3}$ : the main axes of inertia.

the $\alpha_{i}$ are sorted by $\alpha_{1}>\alpha_{2}>\alpha_{3}$.

1. Remark. The $c_{i}$ in Eq. (12) are normalized in the following way: $\Sigma c_{i}=1$ and $0 \leqslant c_{1}, c_{2}, c_{3} \leqslant 1$.

Consequently, the $c_{i}$ can be visualized by a state triangle; see Fig. 3. A high value of $c_{1}$ means that the local structure resembles a cylinder, a high value of $c_{2}$ a plane, and a high value of $c_{3}$ an isotropic structure. The parameters $c_{i}$ can be further used for geometry classification as shown in the following example.

\subsubsection{Example}

We calculate the eigenvectors, eigenvalues, and the $c_{i}$ for the structure from Fig. 4 in the voxel next to the gap. The integration volume is plotted around the voxel.

Table 1 shows how the values of $c_{1}, c_{2}$, and $c_{3}$ change according to the integration size $\delta$. The dominating eigenvector (DEV) changes when the integration size grows and finally they converge as can be seen from Table 1. For small

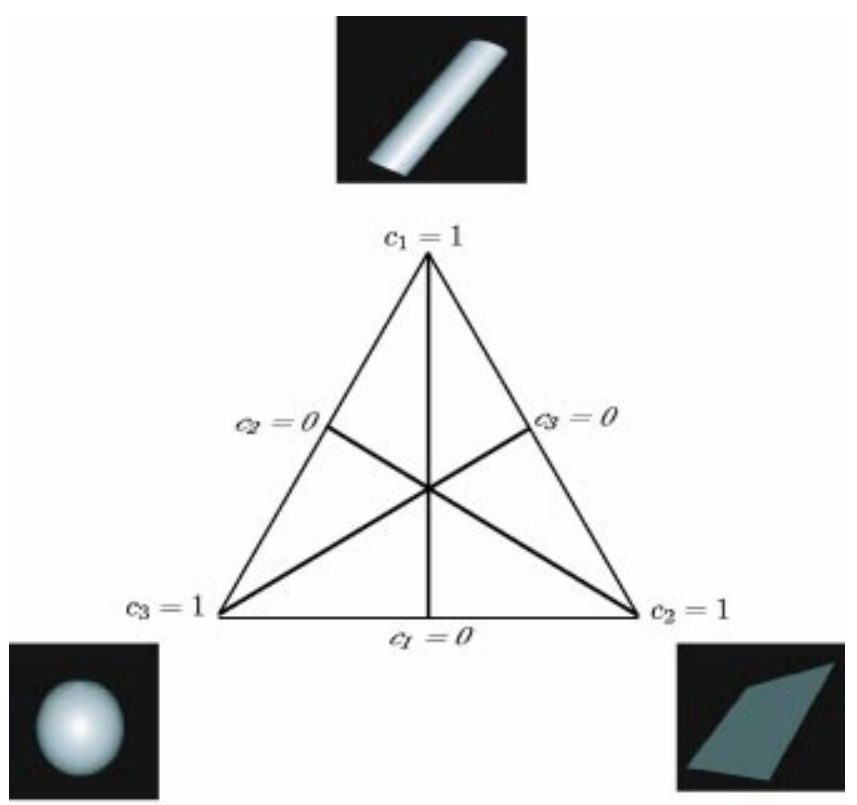

Fig. 3 Visualization of the normalized values $c_{i}$ using a state triangle.

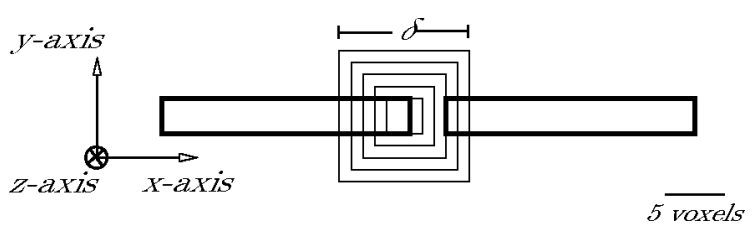

Fig. 4 A simple linear structure to analyze the behavior of the eigenvalues and $c_{i}$ respectively to the scanning range $\delta$. The structure is again assumed to be solid with a constant density value. The structure is three voxel high ( $y$ direction) and three voxel deep ( $z$ direction).

integration volumes the algorithm tends to detect an isotropic structure $(\delta=\{3,5,7\})$ while for integration volumes large enough to reach over the gap $(\delta \geqslant 9)$ a cylindrical structure is identified. Therefore it is important to know the scale of the structure to be detected. Consequently the algorithm can be tuned to detect small structures or large structures. For example, for enhancing dendritic branches we usually choose an integration size of $10 \mu \mathrm{m}$. For enhancement of smaller structures such as dendritic spines a smaller scanning range of about $3 \mu \mathrm{m}$ has to be used.

\subsubsection{Construction of $D(u)$}

We are now in a position to construct the permeability tensor $D(u)$. We define $D(u)$ such that diffusion occurs only parallel to the axis of the tube, but not perpendicular to it. The dominating eigenvector $V_{1}$ of the moment of inertia gives us the local main direction of the structure. So we separate the diffusion direction $\nabla u$ into two, $(\nabla u)_{p}$ and $(\nabla u)_{t}$, with $(\nabla u)_{p} \cdot V_{1}=0$.

Technically speaking this means to transform the vector $\nabla u$ into the eigenspace of the diffusion tensor and multiply the first component with unity, the others with a nearly zero constant $\epsilon$ (which is necessary for well-posedness). So we finally set $D$ from Eq. (5) to:

$$
D(u)=B\left(\begin{array}{ccc}
1 & 0 & 0 \\
0 & \epsilon & 0 \\
0 & 0 & \epsilon
\end{array}\right) B^{T}
$$

with $B=\left(V_{1}, V_{2}, V_{3}\right)$ and $\epsilon>0$ [with $V_{i}$ the eigenvectors; compare with Eq. (10)]. This means that the filter always causes diffusion into the direction of the largest eigenvalue of the local tensor of inertia, even if the local mass is small or nearly isotropic. We found this to be the optimal strategy when preparing neuronal image data for subsequent segmentation (see discussion in Sec. 4). However, for other applica-

Table 1 Dependence of the $c_{i}$ on the integration size $\delta$ in pixels.

\begin{tabular}{cccccc}
\hline scanning range & $\delta=3$ & $\delta=5$ & $\delta=7$ & $\delta=9$ & $\delta=11$ \\
\hline$c_{1}$ & 0.00 & 0.00 & 0.23 & 0.75 & 0.84 \\
$c_{2}$ & 0.53 & 0.00 & 0.00 & 0.00 & 0.00 \\
$c_{3}$ & 0.47 & 1.00 & 0.77 & 0.25 & 0.16 \\
DEV & $y, z$ & $x, y, z$ & $x$ & $x$ & $x$ \\
\hline
\end{tabular}


Nonlinear anisotropic diffusion filtering . . .

tions of the filter it may be advantageous to switch off local diffusion if the data within the scanning range is close to isotropic, or to apply planar diffusion if $c_{2}$ is large.

\subsection{Numerical Solution}

\subsubsection{Discretization and solver}

In order to solve the partial differential equation [Eq. (5)] we use a semi-implicit time discretization and a finite volume spatial discretization. The semi-implicit scheme is advantageous compared to the fully explicit time scheme because the time step size can be increased without losing stability. ${ }^{29}$ For time discretization a simple backward-Euler scheme was used. Starting from

$$
\partial_{t} u=\nabla \cdot(D(u) \nabla u)
$$

we obtain:

$$
\frac{u^{t+1}-u^{t}}{\tau}=\nabla \cdot\left(D\left(u^{t}\right) \nabla u^{t+1}\right)
$$

(where $\tau$ is the time step size without unit). For spatial discretization a finite-volume method was used (see Ref. 30). In this method each voxel is surrounded by a control volume $\Omega_{i}$. The distance between two voxels is $h$. To obtain the discrete nodal value $u_{h, i}^{t+1}$ assigned to voxel $i$, we introduced the following equation:

$$
u_{h, i}^{t+1}=u_{h, i}^{t}+\frac{\tau}{\left|\Omega_{i}\right|}\left\{\sum_{j} u_{h, j}^{t+1} \int_{\partial \Omega_{i}}\left[D\left(u_{h, i}^{t}\right) \nabla \varphi_{j}\right] \cdot \vec{n} \mathrm{~d} s\right\} \forall i
$$

The $\varphi_{j}$ are standard bilinear finite element basis functions. ${ }^{30}$ The integral is approximated by using numerical integration with several integration points $i p_{k}$. Finally we get the linear algebraic equation

$$
(I-\tau A) u_{h}^{t+1}=u_{h}^{t}
$$

with

$$
a_{i j}=\frac{1}{\left|\Omega_{i}\right|} \sum_{k}\left(D\left(u_{h, i}^{t}\right) \nabla \varphi_{j}\left(i p_{k}\right)\right) \cdot \vec{n}\left(i p_{k}\right) \cdot\left|S_{k}\right|,
$$

where $\left|S_{k}\right|$ is the size of the subarea $S_{k} \subset \partial \Omega_{i}$ corresponding to $i p_{k}$. We evaluated the integral by using a standard midpoint rule. Due to the boundary condition in Eq. (16) it is sufficient to consider in Eq. (16) only inner integration points $i p_{k} \in \Omega$ and to ignore boundary integration points $i p_{k} \in \partial \Omega$.

To solve Eq. (17) we used a conjugated gradient (CG) solver (see Ref. 31), which is a standard iterative solver for linear algebraic systems.

\subsubsection{Computational effort}

Because a simple CG solver is used without preconditioner the computational complexity is $\mathcal{O}\left(n^{3 / 2}\right)$ in each time step. To keep the computational effort low we implemented a special algorithm for the computation of the moments. We calculate the moments by using a fast Fourier transform, which transforms the convolution into a multiplication. Another important feature is the convergence of the solver for the linear
Table 2 Convergence of CG solver for a $129^{3}$ voxel image stack (where $\bar{\varrho}$ is the average convergence rate).

\begin{tabular}{lccccccc}
\hline & $\tau=0.2$ & $\tau=0.6$ & $\tau=1.0$ & $\tau=3.0$ & $\tau=5.0$ & $\tau=10.0$ \\
\hline Time [s] & 71 & 111 & 131 & 230 & 300 & 408 \\
Iterations & 6 & 10 & 12 & 22 & 29 & 40 \\
$\bar{\varrho}$ & 0.0319 & 0.1344 & 0.2148 & 0.4304 & 0.5272 & 0.6201 \\
\hline
\end{tabular}

system. Table 2 shows the number of iterations and the time needed to solve the linear algebraic equations up to a defect reduction of $10^{-8}$. The results show the typical increase of number of iterations with increasing $\tau$. However for our computation this increase is not practically significant.

\subsection{In vivo Two-photon Imaging}

Two-photon imaging was performed as described in Ref. 32. Rats were anaesthetized with urethane. A small $(2 \times 2 \mathrm{~mm})$ craniotomy was made over barrel cortex and the dura was removed. The craniotomy was covered with agar (1-1.5\%, type III-A, Sigma) in the following solution (in $\mathrm{mM}$ ): 135 $\mathrm{NaCl}, 5.4 \mathrm{KCl}, 1 \mathrm{MgCl}_{2}, 1.8 \mathrm{CaCl}_{2}, 5 \mathrm{HEPES}$. A glass coverslip was positioned over the agar. This reduced motion of the cortex during recording and imaging.

Neurons were filled with the soluble fluorescent indicator Alexa 594 using the whole-cell patch-clamp technique. Pipettes with 4-6 $\mathrm{M} \Omega$ open tip resistance were filled with the following intracellular solution (in $\mathrm{mM}$ ): $135 \mathrm{~K}$ gluconate, 4 $\mathrm{KCl}, 10 \mathrm{HEPES}, 10 \mathrm{Na}_{2}$-phosphocreatine, $4 \mathrm{Mg}$-ATP, $0.3 \mathrm{Na}$ GTP, $0.2 \%$ biocytin, 0.02 Alexa 594 (pH 7.2; 291-293 mOsm). Recordings were obtained blind, as described in Ref. 33.

Two-photon microscopy was performed using a custombuilt microscope. ${ }^{32}$ The specimen was illuminated with $840-\mathrm{nm}$ light from a pulsed Ti:sapphire laser with a repetition rate of $80 \mathrm{MHz}$ and 100- to 150-fs pulse width (Mira 900, Coherent). Excitation light was focussed onto the specimen using a $40 \times$, NA 0.8 water immersion objective (Zeiss).

Emitted fluorescence was deflected by 680-nm LP dichroic mirror and detected with a photomultiplier tube (Hamamatsu). An infrared-blocking filter (Calflex, Linos) and an emission filter (HQ 610/75M, Chroma) were used in the detection pathway. Scanning and image acquisition were controlled using custom software (R. Stepnoski and M. Müller, Lucent Technologies, New Jersey and MPImF, Heidelberg).

\section{Results}

\subsection{Testing the Filter on Artificial Data}

We designed a simple data stack containing a Y-like structure to test how the filter works under controlled conditions. In Fig. 5(a) one slice of this $65^{3}$ voxel data stack is illustrated. The Y has three gaps, each of them being three voxels wide.

We applied the anisotropic diffusion filter to this data set, with a scanning range of 10 voxels [Fig. 5(b)]. As expected the filter preserved the diameters of the $\mathrm{Y}$ branches but filled in the gaps. For comparison we also applied a simple isotropic diffusion to the same data set [Fig. 5(c)]. Although the linear 

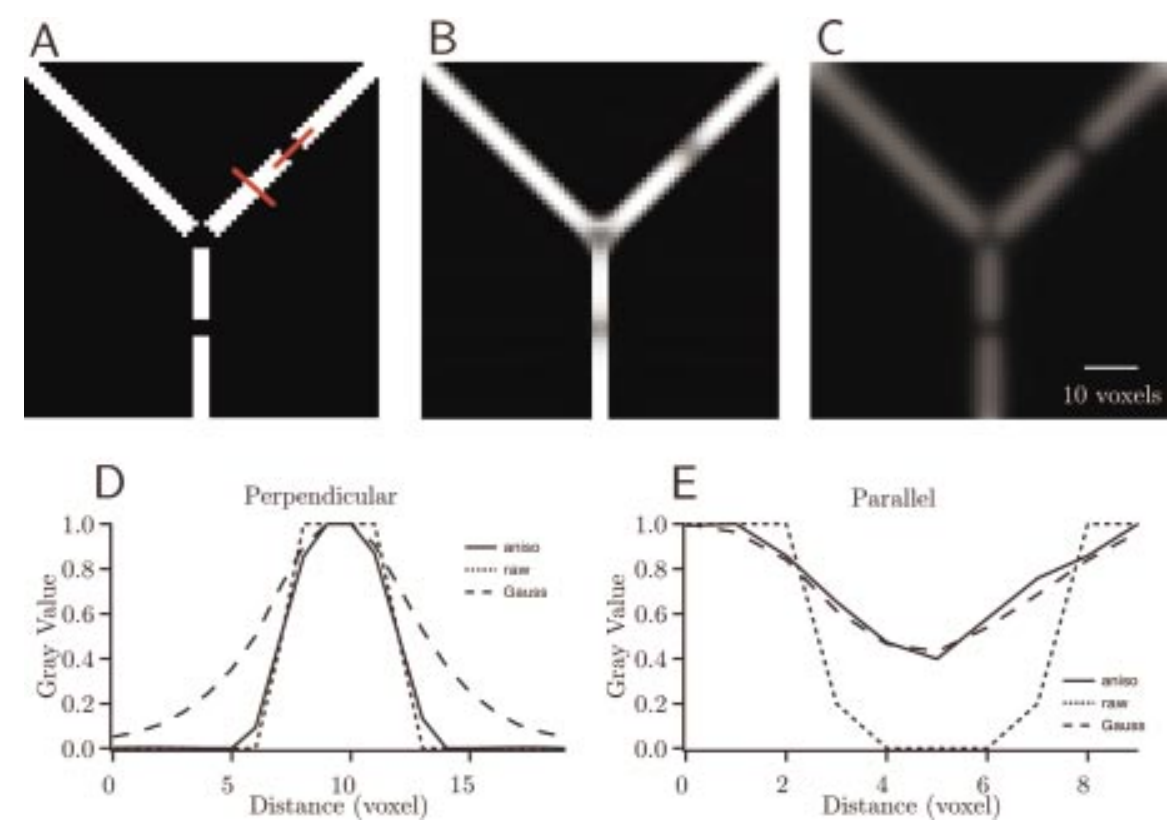

Fig. 5 Test data stack. A simple test data stack with a Y-like structure was created to analyze the behavior of the filter. The structure is assumed to be solid with a density value of 1 . The diameter of the Y-like structure is three voxels. The background is defined with density value zero. The structure has three gaps, each of which is three voxels wide. (a) One slice of the initial data. The red lines mark the area where the data for the signal profiles shown in (d), (e) were taken from. (b) Nonlinear anisotropic diffusion. (c) Linear isotropic diffusion. The normalized signal profiles demonstrate the effect of the anisotropic filter and the Gaussian smoothing. (d) Signal perpendicular to the branch. Anisotropic filtering conserves the signal profile. However, Gaussian blurring distorts the signal structure. Sharp edges are lost. (e) Signal along the branch. Both filters fill the gap in nearly the same way. 

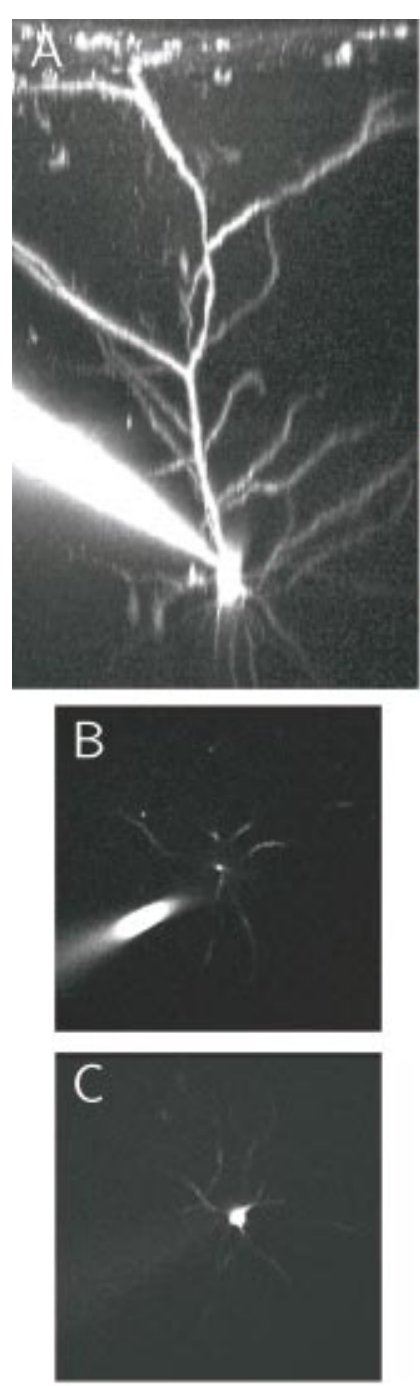
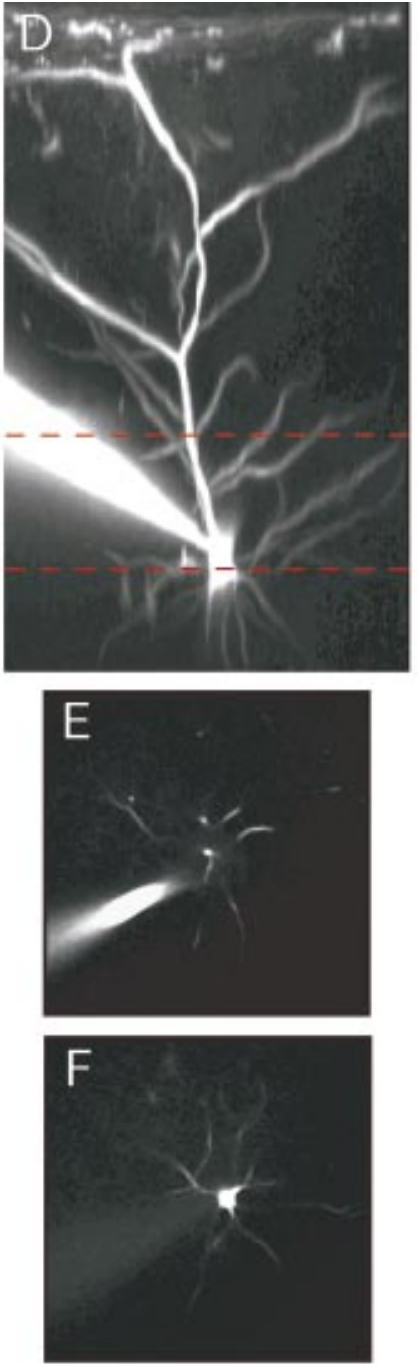
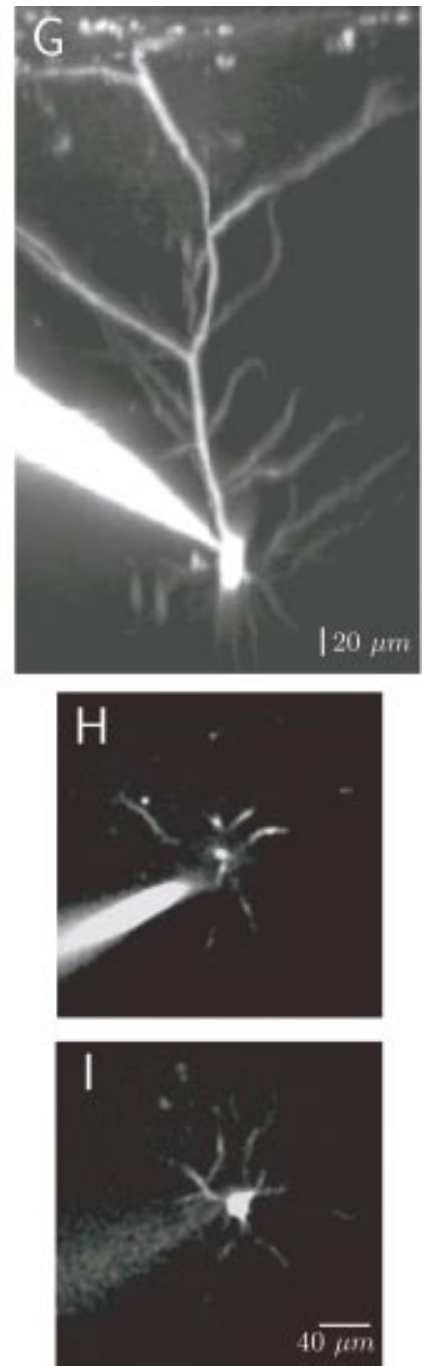

Fig. 6 A layer 2/3 pyramidal neuron was imaged using two-photon microscopy. The data stack consists of 216 slices each $2 \mu \mathrm{m}$ thick. Each slices has $256^{2}$ pixels. One pixel is $1 \mu \mathrm{m}$ in $x$ direction and $1 \mu \mathrm{m}$ in $y$ direction. (a) Sagittal view of a maximum intensity projection of the raw data. (b), (c) Two slices of the raw data. The loading pipette is visible on the left side of the images. (d), (e), (f) Same data after anisotropic diffusion filtering. The broken red lines in (d) indicate the depth where the two slices shown are taken from. In (g), (h), (i) a Gaussian blurring is applied to the raw data.

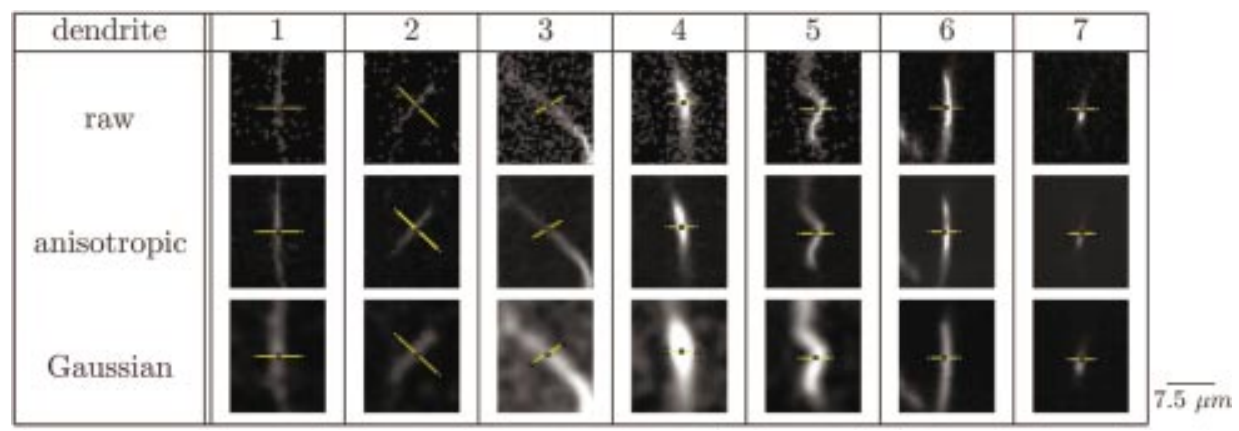

Fig. 7 Close-up views of dendritic branches. The conservation of the diameter of the dendrites was one of the major goals for the design of the filter. In this figure the dendrites which were used to measure the change in the diameter are shown. See Table 3 for results of the measurement of the full width at half maximum. 
Table 3 Changes in diameter (FWHM) after anisotropic diffusion filtering or Gaussian blurring compared to raw size (in $\mu \mathrm{m}$ ): $\mathrm{f} / \mathrm{raw}$ is the ratio of the FWHM after anisotropic diffusion to the raw FWHM, and G/raw is the ratio of the FWHM after Gaussian blur to the raw FWHM. Seven different dendrites have been evaluated.

\begin{tabular}{lccccccccc}
\hline dendrite & 1 & 2 & 3 & 4 & 5 & 6 & 7 & mean & s.d. \\
\hline raw & 1.45 & 0.65 & 2.15 & 1.25 & 1.3 & 2.05 & 2.3 & & \\
filtered & 1.35 & 1.25 & 1.55 & 1.0 & 0.95 & 1.35 & 1.5 & & \\
Gauss & 2.1 & 2.25 & 2.95 & 2.3 & 2.35 & 2.8 & 2.65 & & \\
f/raw & 0.93 & 1.92 & 0.72 & 0.80 & 0.73 & 0.66 & 0.65 & 0.92 & 0.45 \\
G/raw & 1.45 & 3.46 & 1.37 & 1.84 & 1.81 & 1.37 & 1.15 & 1.78 & 0.78 \\
\hline
\end{tabular}

filter closed the gaps similar to the anisotropic filter, the diameters of the branches were severely broadened. Because of the enlargement of the structure and the conservation of the mean gray value the peak signal level of the isotropically filtered image is reduced compared to the anisotropic case.

To further quantify the differences between anisotropic and isotropic filtering, we measured the spatial intensity profiles parallel and perpendicular to one branch [Figs. 5(d) and 5(e)]. Broadening of this structure was analyzed by calculating the full width at half maximum (FWHM) for the raw data set, and for the anisotropically and isotropically filtered data set, respectively (using linear interpolation). The anisotropic filter maintained the half width of the branch $\left(\mathrm{FWHM}_{\mathrm{raw}}=6\right.$, $\mathrm{FWHM}_{\text {anisotropic }}=5.84$, measured in pixels). In contrast the FWHM was increased with linear filtering $\left(\mathrm{FWHM}_{\text {gauss }}\right.$ $=6.91$.). Because of the step-like nature of the artificial data the anisotropic diffusion even caused a slight reduction of the FWHM. The spatial profiles parallel to the branch demonstrate how both filters fill the gap in nearly the same way [Fig. $5(\mathrm{e})]$. This is expected because along detected linear structures anisotropic diffusion and Gaussian blurring convolve the data nearly equally.

\subsection{Testing the Filter on Two-photon Images}

We next applied the anisotropic diffusion algorithm to 3-D fluorescence image stacks of pyramidal neurons in rat neocortex obtained using two-photon microscopy. ${ }^{6}$ Neurons were filled with a fluorescent dye via the whole-cell patch pipette. The data typically consisted of several hundreds of slices taken at $2 \mu \mathrm{m}$ focal increments, each consisting of 256 $\times 256$ pixels (8-bit depth). A maximum intensity side projection of a raw data set is shown in Fig. 6(a). In addition, two example slices from different focal planes are shown [Figs. 6(b) and 6(c)] to illustrate how noisy the raw images are. In particular, thin basal dendrites close to the soma are difficult to resolve. Following anisotropic diffusion the signal-to-noise ratio was improved and the thin dendrites can easily be separated from background [Figs. 6(d)-6(f)]. To gain an impression of how the filter works we again compared the filter result to the Gaussian blur [Figs. 6(g) $-6(\mathrm{i}) ; \sigma=2.8$ voxel]. The difference between isotropic and anisotropic filtering is particularly evident at the basal dendrites. After Gaussian filtering it is nearly impossible to see thin dendrites in slices deep inside the tissue, whereas the anisotropic diffusion further enhances the signal-to-noise ratio of these fine structures.

For automatic reconstruction of the dendritic tree it is very important how dendritic diameters are affected by the filter. To quantify the diameters we again measured the FWHM of seven dendrites of different sizes. Some of them were parallel to the coordinate axis, some were oblique (see Fig. 7). The effects of these filters are shown in Table 3. The anisotropic filter has little effect on dendritic diameter (on average they shrink by a factor of $0.92 \pm 0.45$ ) whereas Gaussian blurring almost doubles the diameter on average (expansion by a factor of 1.78 \pm 0.78). In Fig. 9(a) the (normalized) signal profile of dendrite number 5 is shown.

We next examined whether the filter bridges gaps in twophoton microscopic data. Therefore we first enlarged one slice of the data set and measured the signal profile along one dendrite. In Fig. 8(a) the same raw data as in Fig. 6 are shown on an expanded scale. Figure 8(b) shows the data after anisotropic filtering. The box indicates the areas which are presented in Figs. 8(c) and 8(d). In Fig. 8(c) there are discontinuities in the dendritic branch as bright pixels are separated by dark pixels. After anisotropic filtering these discontinuities are closed [see Figs. 8(d) and 9(b)]. The filter effect is clearer when looking at a projection. Figures $8(\mathrm{e})$ and $8(\mathrm{f})$ are maximum intensity projections of 10 slices. Dendrites, which were difficult to follow in the raw data set, are easy to find in the filtered data, for instance, the dendrite in the upper left corner.

\subsection{Application to Binary Data}

One of the purposes of the filter is to preprocess the data for segmentation of objects. Even with this preprocessing some gaps may remain after a segmentation process. In this case it is convenient to apply the anisotropic diffusion filter a second time on the segmented (i.e., binary) data. An example application of the filter on binary data is shown in Fig. 10. The filter bridges the remaining gaps while preserving the diameter of the dendrites.

To illustrate how the filter facilitates automatic reconstruction of the dendritic morphology, we compared reconstructions of the same layer $2 / 3$ pyramidal neuron without and with prior filtering (Fig. 11). Filtering included a first pass of anisotropic diffusion filtering on the raw data, subsequent segmentation, and a second pass of the filter on the segmented 

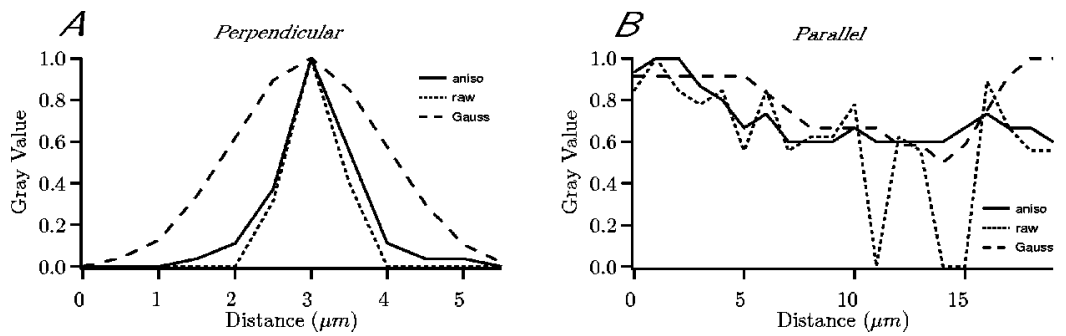

Fig. 9 Conservation of dendritic diameters and closure of gaps in the two-photon fluorescence data stack. The plot in (a) shows the normalized signal profile perpendicular to dendrite 5 in Fig. 7. Again the anisotropic diffusion filtering conserves the signal profile whereas the Gaussian blurring causes a widening of the signal. In (b) the closing of a gap is demonstrated by showing the (normalized) signal along the dendrite marked in Fig. 8(a) by red arrows. There is nearly no difference between anisotropic diffusion and blurring.

(binary) data. The unfiltered and filtered data were then both segmented, reconstructed using the same algorithm and parameter settings, and visualized using the NEURON simulation environment. ${ }^{24}$ In both reconstructions the main apical dendrites are well detected. However, the fine basal dendrites and the thin side branches of the neuron are missing in the case of no pre-processing [Fig. 11(a)] whereas these structures are present following application of the anisotropic diffusion filter [Fig. 11(b)]. This is particularly evident for the fine basal dendrites deep within the neocortex. A detailed discussion of results obtained with different reconstruction procedures will be presented in a forthcoming paper.

\section{Discussion}

We have presented a method for filtering two-photon microscopy data of neuronal morphologies. The method is based on anisotropic diffusion in three dimensions. The key idea of the algorithm is the use of the local moments of inertia to reliably detect the dimensionality (isomorph, planar, or linear) and the orientation of the morphological structures, particularly of dendrites. The filter is controlled by adapting the diffusion tensor in a way to produce smoothing along but not perpendicular to the structure. We have demonstrated that anisotropic filtering preserves dendritic diameters in a set of artificial test data and in two-photon microscopic images of neocortical neurons. In addition it bridges apparent gaps in images of dendrites, which result from poor signal-to-noise ratio, by smoothing along the dendritic axis similar to a Gaussian blurring filter. It should be noted that dendrite diameters can only be resolved accurately if they are larger than the optical resolution of the microscope system. This is a fundamental limitation, which is not overcome by anisotropic diffusion filtering. For thin dendrites the diameters obtained therefore represent an overestimate of the true diameters because they are convolved with the point spread function of the microscope. However, in any case anisotropic diffusion filtering does not introduce an additional systematic bias in dendritic diameters.

The anisotropic diffusion filter was implemented using a semi-implicit scheme for time discretization and a finite-
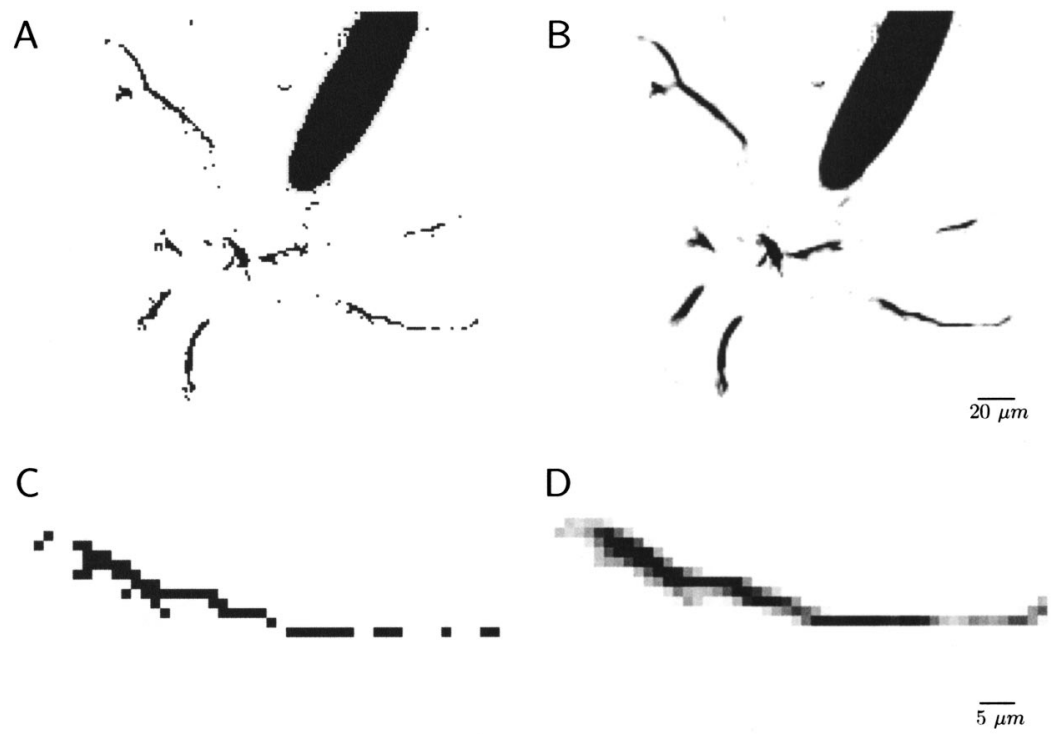

Fig. 10 Nonlinear anisotropic filter applied to binary data. During the reconstruction process filtering on binary data is needed. These figures illustrate how the filter can be used on binary data, too. The initial binary data was obtained from the same raw data as used in Fig. 6 . (a) Initial binary data. The data was filtered by nonlinear anisotropic diffusion with $\tau=2.0$, two time steps, and $\delta=10 \mu \mathrm{m}$. The result is shown in (b). The same region as in Fig. 6 was chosen for analyzing the data at higher magnification (c), (d). The gaps in (c) are closed in (d). The diameter is nearly unchanged. 

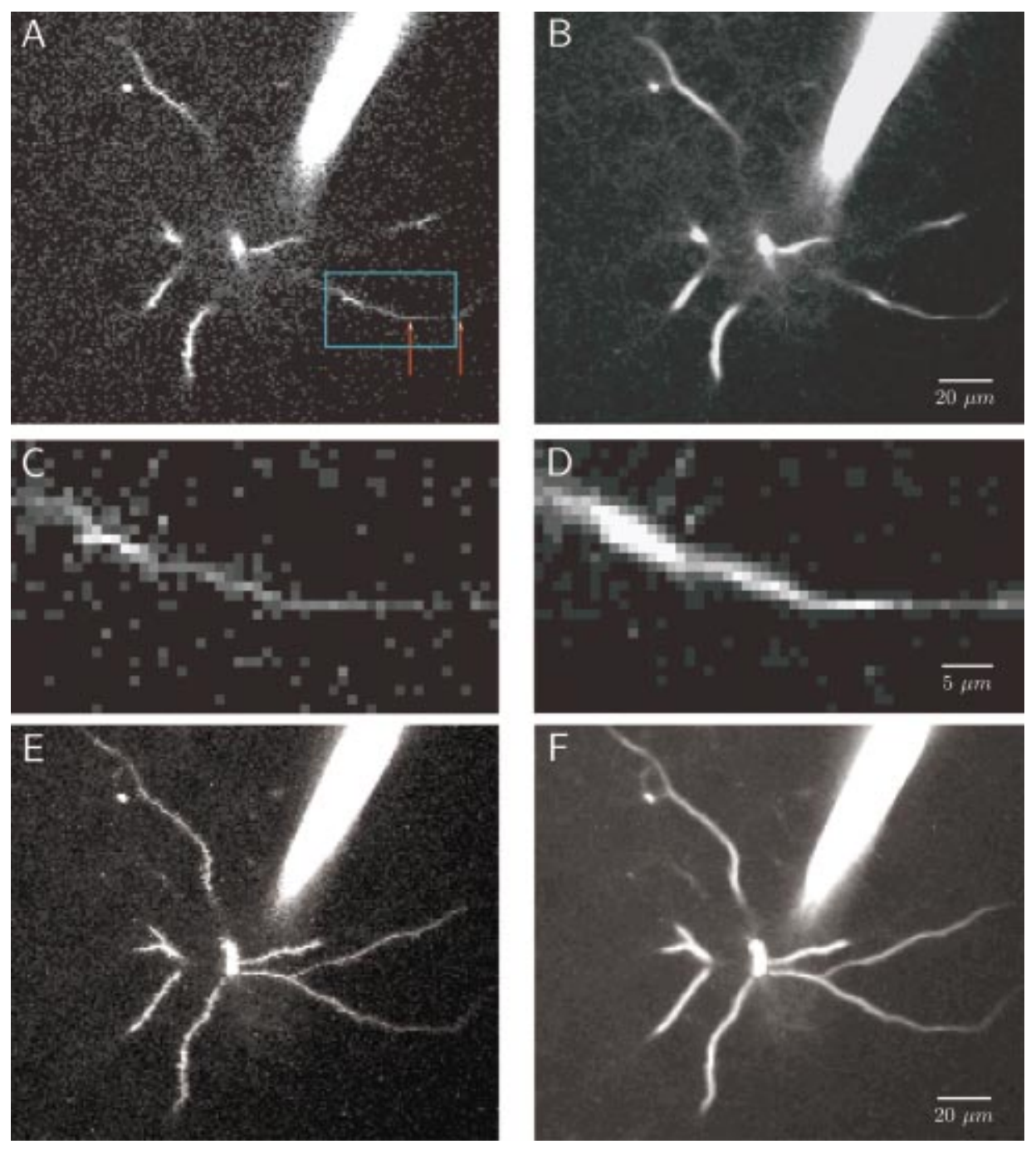

Fig. 8 Zooming into one slice illustrates further how the filter enhances the quality of the data. One slice of the raw data from Fig. 6 is presented in (a). The red arrows mark the line where the signal profiles along the dendrite [see Fig. 9(b)] were measured. The blue box indicates the area from which panel (c) and panel (d) respectively were taken from. (b) Data after nonlinear anisotropic diffusion filtering. (c) and (d) Initial data (filtered data) at higher magnification. (e) and (f) Maximum intensity $z$ projections of ten slices of the raw and the filtered data respectively are illustrated. 

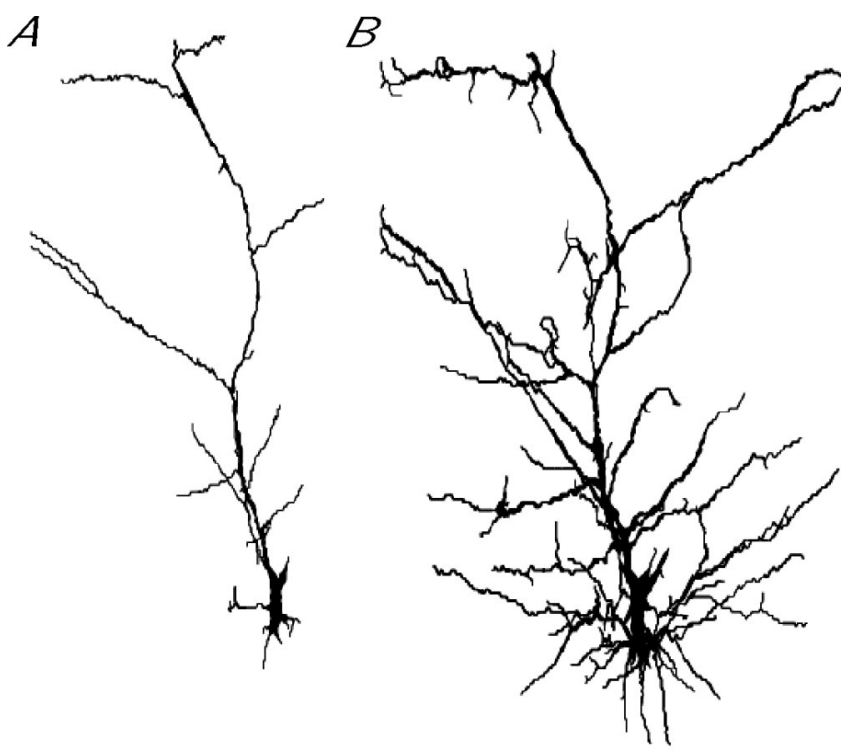

Fig. 11 Automatic reconstruction of neuronal morphology from twophoton fluorescence data (same layer 2/3 pyramidal neuron as in Fig. 6). The morphology of soma and dendrites is presented using the simulation program NEURON. ${ }^{24}$ (a) Result of segmentation and reconstruction without prior anisotropic diffusion filtering. (b) Result of filtering, segmentation, and a second pass of the filter over the segmented (binary) data, followed by the same segmentation and reconstruction algorithms with the same parameter settings as in (a).

volume method for spatial discretization. The semi-implicit time scheme is advantageous since each time step can be used with a larger time step size without losing stability. ${ }^{29}$ In contrast, explicit schemes for time disretization are limited in the time step size by the Courant-Friedrich-Levy condition. ${ }^{29}$ Smaller time steps have to be used so that the number of time steps required to achieve the same filtering increases. As a result the time to solution is typically longer for an explicit scheme compared to an implicit scheme. Due to the complexity of the filtering algorithm, anisotropic diffusion filtering of large data sets is time consuming. But in some parts of the algorithm parallelization is possible, for instance for the calculation of the tensor of inertia or the assembly of the stiffness matrix [see Eq. (17)]. In the future, such parallelization will substantially reduce the computation time.

Anisotropic diffusion filtering offers a convenient way to enhance the quality of fluorescence image stacks without using information about the microscope system used for imaging. The signal-to-noise ratio is enhanced such that the differentiation between foreground and background (segmentation) can easily be done by a simple local thresholding algorithm. The smooth signal profile along structures and the large gradient between structure and background allow even the detection of small dendrites several hundred micrometers deep inside the cortex. Anisotropic diffusion filtering therefore represents an excellent starting point for automatic reconstruction of neurons. In addition, filtering and segmentation can be iterated since the filter does not depend on the depth of the data. It works both on grayscale and on binary data. The advantage of our method compared, for instance, to the method of wavelet shrinkage is that no a-priori information about the given data set is needed. Thus, for a specific struc- ture a general filter algorithm can be used, offering the possibility of automated filtering of large numbers of data sets. Nevertheless it will be interesting to compare the present approach to the wavelet shrinkage method used in Ref. 21. Mrázek et al. ${ }^{34}$ showed for the one-dimensional case that the Perona-Malik diffusivity or the Weickert diffusivity have a corresponding wavelet shrinkage. This means that certain diffusion processes can be described by wavelet shrinkage. In the future it may be possible to extend this connection between anisotropic diffusion filtering and wavelet transformations to the three-dimensional case.

For those who are interested in trying the anisotropic diffusion filter by themselves we offer a sample program in Ref. 35.

We are currently developing a new software toolbox, NEURA, designed for automatic reconstruction of neuronal morphologies, which includes the anisotropic diffusion for data preprocessing. Preliminary results indicate that the combination of the anisotropic filter with a sophisticated segmentation algorithm can deliver a good restoration of the neuron shape. Even fine dendrites can be tracked using graph tracking algorithms, yielding a reconstruction of the branching pattern of the neuron. Here the next challenge will be to apply the reconstruction algorithm to the finer axonal arborisation of cortical neurons. The overall algorithm for neuron reconstruction implemented in NEURA will be discussed in a forthcoming paper.

\section{Acknowledgments}

We are grateful to Professor M. Rumpf for the initial help. This project is supported by Leica Microsystems, Mannheim. Thanks to C. Reisinger for the help with software development and P. Frolkovic for stimulating discussions on solving partial differential equations.

\section{References}

1. W. Denk, J.H. Strickler, and W.W. Webb, "Two-photon laser scanning fluorescence microscopy," Science 248, 73-76 (1990).

2. W. Denk and K. Svoboda, "Photon upmanship: why multiphoton imaging is more than a gimmick," Neuron 18, 351-357 (1997).

3. F. Helmchen and W. Denk, "New developments in multiphoton microscopy," Current Opinion Neurobiol. 12, 593-601 (2002).

4. W.R. Zipfel, R.M. Williams, and W.W. Webb, "Nonlinear magic: multiphoton microscopy in the biosciences," Nat. Biotechnol. 21, 1369-1377 (2003).

5. P. Theer, M.T. Hasan, and W. Denk, "Two-photon imaging to a depth of 1000 micron in living brains by use of a ti:al2o3 regenerative amplifier," Opt. Lett. 28, 1022-1024 (2003).

6. K. Svoboda, W. Denk, D. Kleinfeld, and D.W. Tank, "In vivo dendritic calcium dynamics in neocortical pyramidal neurons," Nature (London) 385, 161-165 (1997).

7. F. Helmchen and J. Waters, "Ca2 + imaging in the mammalian brain in vivo," Eur. J. Pharmacol. 447, 119-129 (2002).

8. J. Kim, T. Dittgen, A. Nimmerjahn, J. Waters, V. Pawlak, F. Helmchen, S. Schlesinger, P.H. Seeburg, and P. Osten, "Sindbis vector sinrep(nsp2s726): a tool for rapid heterologous expression with attenuated cytotoxicity in neurons," J. Neurosci. Methods 133, 81-90 (2004).

9. B. Lendvai, E.A. Stern, B. Chen, and K. Svoboda, "Experiencedependent plasticity of dendritic spines in the developing rat barrel cortex in vivo," Nature (London) 404, 876-881 (2000).

10. G. Feng, R.H. Mellor, M. Bernstein, C. Keller-Peck, Q.T. Nguyen, M. Wallace, J.M. Nerbonne, J.W. Lichtman, and J.R. Sanes, "Imaging neuronal subsets in transgenic mice expressing multiple spectral variants of gfp," Neuron 28, 41-51 (2000).

11. F. Helmchen, K. Svoboda, W. Denk, and D.W. Tank, "In vivo den- 
dritic calcium dynamics in deep-layer cortical pyramidal neurons," Nat. Neurosci. 2, 989-996 (1999).

12. D. Jaeger, "Accurate reconstruction of neuronal morphology," in Computational Neuroscience: Realistic Modeling for Experimentalists, pp. 159-178, CRC Press, Boca Raton, Florida (2001).

13. A. Dima, M. Scholz, and K. Obermayer, "Automatic 3d-graph construction of nerve cells from confocal microscopy scans," J. Electron. Imaging 12(1), 134-150 (2003).

14. A. Dima, M. Scholz, and K. Obermayer, "Automatic segmentation and skeletonization of neurons from confocal microscopy images based on the 3-d wavelet transform," IEEE Trans. Image Process. 11(7), 790-801 (2002).

15. A. Rodriguez, D. Ehlenberger, K. Kelliher, M. Einstein, S.C. Henderson, J. Morrison, P. Hof, and S. L. Wearne, "Automated reconstruction of three-dimensional neuronal morphology from laser scanning microscopy images," Methods 30, 94-102 (2003).

16. M. Maravall, I.Y.Y. Koh, W.B. Lindquist, and K. Svoboda, "Experience-dependent changes in basal dendritic branching of layer $2 / 3$ pyramidal neurons during a critical period for developmental plasticity in rat barrel cortex," Cereb. Cortex 14, 655-664 (2004).

17. R.C. Gonzales and P. Wintz, Digital Image Processing, AdisonWesley (1987).

18. J. Weickert, Anisotropic Diffusion in Image Processing, Teubner, Stuttgart (1998)

19. B. Jaehne, "Image sequence analysis of complex physical objects: nonlinear small scale water surface waves," IEEE Computer Society Press, pp. 191-200 (1987).

20. D.L. Donoho and I.M. Johnstone, "Ideal spatial adaptation via wavelet shrinkage," Biometrika 81, 425-455 (1994).

21. A. Dima, M. Scholz, and K. Obermayer, "Semi-automatic quality determination of $3 \mathrm{~d}$ confocal microscope scans of neuronal cells denoised by 3d-wavelet shrinkage," Proc. SPIE 3723, 446-457 (1999).

22. P. Perona and J. Malik, "Scale space and edge detection using anisotropic diffusion," Proc. IEEE Comp. Soc. Workshop on Computer
Vision, Miami Beach, Nov. 30-Dec. 2, 1987, IEEE Computer Society Press, Washington, D.C. (1987).

23. F. Lenzen, "3D-Rekonstruktion von DNA-Strukturen," Master's thesis, Universität Bonn (2001).

24. M.L. Hines and N.T. Carnevale, "The neuron simulation environment," Neural Comput. 9, 1179-1209 (1997).

25. O. Forster, Analysis III, Vieweg, Braunschweig (1996).

26. E. Baensch and K. Mikula, "Adaptivity in $3 \mathrm{~d}$ image processing," Comput. Visualization Sci. 4, 21-30 (2001).

27. H. Gajewski and K. Gaertner, "On a nonlocal model of image segmentation," preprint, Weierstrass-Institut fuer Angewandte Analysis und Stochastik (2002).

28. U. Clarenz, M. Rumpf, and A. Telea, "Robust feature detection and local classification for surfaces based on moment analysis," IEEE Trans. Visualization Comput. Graph. (submitted for publication).

29. H.R. Schwarz, Numerische Mathematik, Teubner, Stuttgart (1997).

30. W. Hackbusch, "On first and second order box schemes," Computing 41, 277-296 (1989).

31. W. Hackbusch, Iterative Loesung grosser schwachbesetzter Gleichungssysteme, Teubner, Stuttgart (1993).

32. J. Waters, M. Larkum, B. Sakmann, and F. Helmchen, "Supralinear $\mathrm{Ca}^{2+}$ influx into dendritic tufts of layer $2 / 3$ neocortical pyramidal neurons in vitro and in vivo," J. Neurosci. 23(24), 8558-8567 (2003).

33. T.W. Margrie, M. Brecht, and B. Sakmann, "In vivo, low-resistance, whole-cell recordings from neurons in the anaesthetized and awake mammalian brain," Pfluegers Archiv. 444, 491-498 (2002).

34. P. Mrázek, J. Weickert, and G. Steidl, "Correspondences between wavelet shrinkage and nonlinear diffusion," Scale-Space 2003, Lecture Notes in Computer Science, Vol. 2695, pp. 101-116, Springer (2003).

35. ANISOFILTER, http://www.mpimf-heidelberg.mpg.de/ pbroser. 